\title{
Not Just Fat: Imaging in Abdominal Fat Pathology
}

\author{
Aruna R. Patil ${ }^{1}$ Shrivalli Nandikoor ${ }^{1}$ Pramod Jagannath ${ }^{2}$ Amit Bansal ${ }^{2}$
}

${ }^{1}$ Department of Radiology, Apollo Hospitals, Bengaluru, Karnataka, India

${ }^{2}$ Department of Gastrointestinal, HPB and Minimal Access Surgery, Apollo Hospitals, Bengaluru, Karnataka, India

J Gastrointestinal Abdominal Radiol ISGAR 2021;4:49-57.

\begin{abstract}
Address for correspondence Aruna R. Patil, MD, DNB, FRCR, Department of Radiology, Apollo Hospitals, Bengaluru 560076, Karnataka, India (e-mail: dr.arunarpatil@gmail.com).
\end{abstract}

\begin{abstract}
Keywords

- omentum

- computed tomography

- ultrasonography

- infarction

- appendagitis

Fat in abdomen has diverse distribution and function. Insult to fat due to several causes can result in infarction or necrosis and present as acute abdomen clinically. Intra-abdominal focal fat infarction is one such condition that comprises of epiploic appendagitis, perigastric appendagitis, omental infarction, and torsion of fatty appendage of falciform ligament that have characteristic imaging features. Secondary causes of fat necrosis include pancreatitis or trauma related. Metabolic or responsive fat changes, like hypertrophy and dystrophy, can be diagnosed on imaging especially on computed tomography. Mesentric fat stranding including the mesentric panniculitis spectrum poses diagnostic dilemma and the causes and imaging role are covered in this review. Some infections and neoplasms that preferably affect abdominal fat compartments may mimic benign conditions, although some have specific patterns of involvement.
\end{abstract}

\section{Introduction}

Abdominal fat can be divided into intra- and extraperitoneal compartments. Fat within the omentum, mesentry, and mesocolon constitute the intraperitoneal compartment and fat in retroperitoneal, preperitoneal, and abdominal wall constitute extraperitoneal compartment. ${ }^{1}$ The conditions involving the abdominal fat pathology include intra-abdominal focal fat infarct (IFFI), metabolic or reparative response, infective, and neoplastic. Some of the fat pathology cause acute abdomen and imaging knowledge is essential for correct diagnosis and hence appropriate management. - Table 1 summarizes the causes.

\section{Role of Imaging}

Imaging plays a vital role and sometimes the only means of diagnosis. Ultrasonography (USG) is usually the initial modality as most of the fat infarcts present with acute abdomen. ${ }^{2}$ However, it is user dependent and of limited utility in obese patients. Abdominal fat is variably echogenic on USG with inflamed fat appearing more echogenic with probe tenderness if superficial. Computed tomography (CT) is a very useful and preferred modality. Early fat changes can

published online October 22, 2020
DOI https://doi.org/

$10.1055 / \mathrm{s}-0040-1718251$ ISSN 2581-9933. be confidently picked up and characterized. Use of intravenous contrast should be tailored to the clinical presentation. Magnetic resonance imaging (MRI) is generally not used for diagnosis of inflammatory causes and is reserved for tumor characterization and extent and as a problem-solving tool. Visceral fat quantification, a marker of insulin resistance and dyslipidemia is possible using MRI software. ${ }^{3}$ Positron emission tomography-computed tomography (PET-CT) has limited role, but F-18 fluorodeoxyglucose (FDG) uptake is noted in mesentric panniculitis and sclerosing mesenteritis mimicking neoplasm. ${ }^{4}$

\section{Intra-abdominal Focal Fat Infarction}

This is an umbrella term comprising of conditions where the principle cause is infarction of fatty tissue with or without torsion. The fatty structure undergoes ischemia due to vascular compromise and additionally gets inflamed. The conditions that are included are epiploic appendagitis, perigastric appendagitis, omental infarct, and torsion of fatty appendage of falciform ligament. ${ }^{2}$ Acute abdominal pain is the most common presentation and these conditions easily mimic potential surgical conditions such as appendicitis and diverticulitis. 


\section{Epiploic Appendagitis}

Appendages epiploicae are fat containing peritoneal outpouchings, arising from the colon containing central vascularity ( - Fig. 1). Rectum is generally spared. Their long-shape predisposes to torsion resulting in infarction of the fat typically presenting with acute focal pain. This condition predominates in fifth decade, shows male predilection and commonly involves sigmoid colon. ${ }^{5}$ If right sided, it mimics

Table 1 Causes of abdominal fat abnormalities

\begin{tabular}{|l|}
\hline Causes of abdominal fat abnormalities \\
\hline Intra-abdominal focal fat infarction \\
\hline a. Epiploic appendagitis \\
b. Perigastric appendagitis \\
c. Omental infarct: primary and secondary \\
d. Encapsulated fat necrosis \\
e. Torsion of fatty appendage of falciform ligament \\
\hline Secondary fat saponification \\
\hline Metabolic, reparative, and responsive causes \\
\hline a. Lipohypertrophy \\
b. Lipomatosis: generalized and focal \\
c. Lipodystrophy \\
d. Dercum's disease \\
\hline Misty mesentry: mesentric panniculitis spectrum \\
\hline Infections \\
\hline Neoplasms \\
\hline
\end{tabular}

appendicitis clinically. USG is the initial modality of choice with infarcted fat appearing as tender focal echogenicity (-Fig. 2). Echogenic fat points toward abnormality and is referred to as "sentinel sign." ${ }^{6}$ It is not a specific sign of appendagitis but presence of tender echogenic fat warrants further evaluation of underlying cause which could be a pure fat abnormality or secondary to visceral inflammation. On CT, appendagitis appears as oval/linear fat containing structure with hyperattenuating rim and central vessel with dominant periappendageal inflammation. 5,7,8 "Hyperattenuating ring sign" can be used as a primary diagnostic criterion. ${ }^{7}$ The central venous pedicle thrombosis within the inflamed appendage is referred to as the "central dot sign." features are generally classical for the diagnosis (-Fig. 3). Fat stranding secondary to diverticulitis is a close differential,
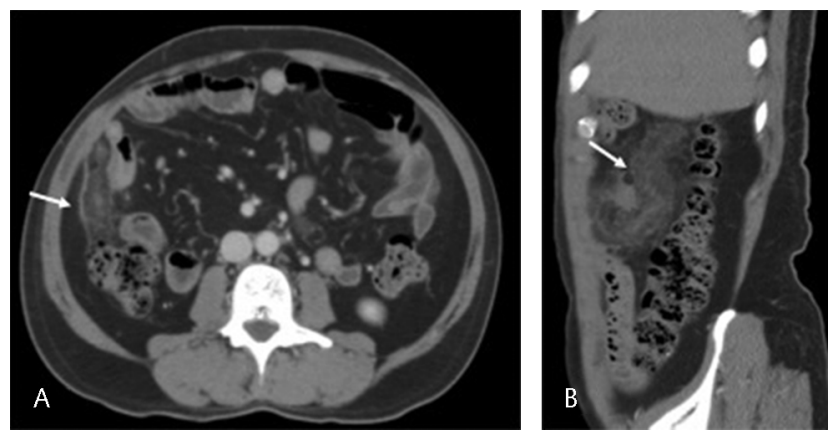

Fig. 3 CT in axial (A) and sagittal plane (B): linear fat density with central vessel related to right colon. Note peripheral hyperattenuating rim (arrow)-classical epiploic appendagitis. CT, computed tomography.
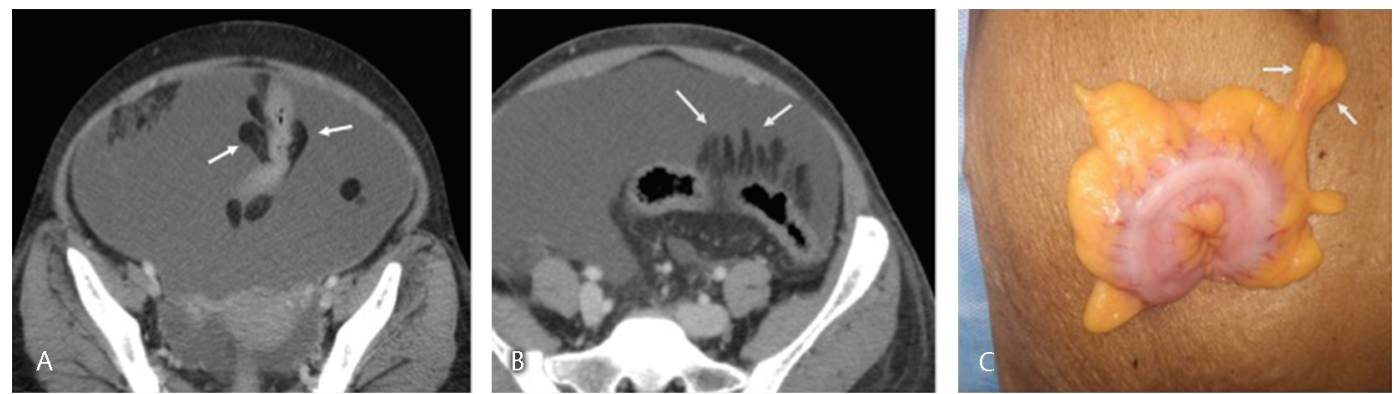

Fig. 1 (A, B) CECT axial sections in a patient with gross ascites shows normal linear fatty appendages from the sigmoid colon (arrows). Presence of ascites make the structures stand out. (C). Intraoperative appearance of normal sigmoid epiploic appendages. CECT, contrast enhanced computed tomography.
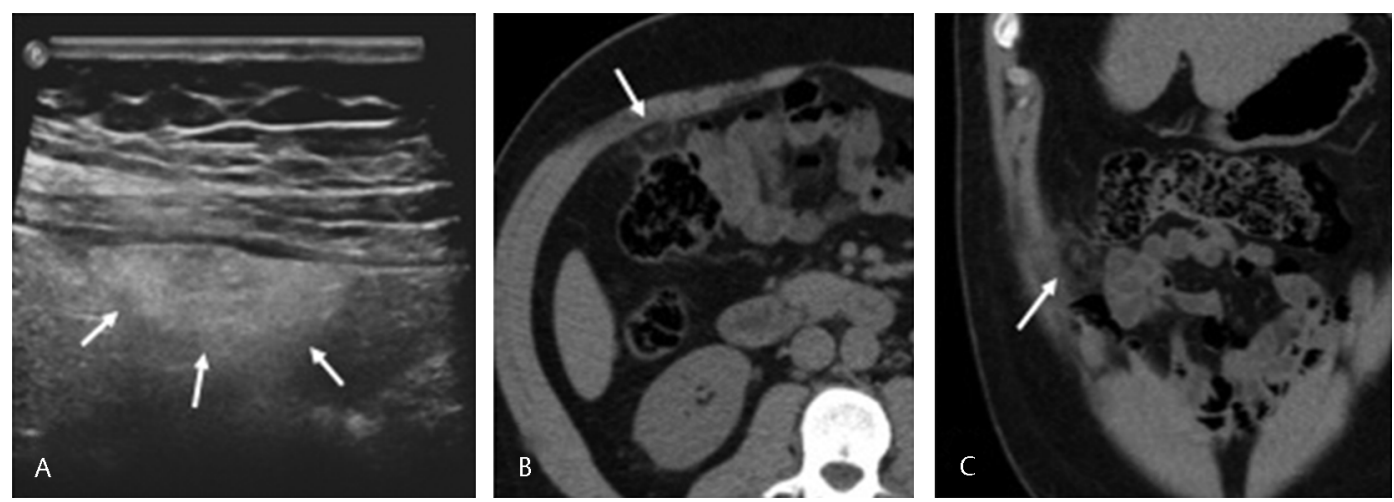

Fig. 2 A 38-year-old male with focal hypochondriac tenderness. (A). USG of right upper abdomen shows superficial subparietal focal echogenic area (arrows) with probe tenderness. (B, C) Plain CT in axial and coronal planes at same level shows focal fat inflammation with central vessel related to right colon (arrows) consistent with epiploic appendagitis. CT, computed tomography; USG, ultrasonography. 
and careful look for an inflamed diverticulum and presence of diverticulosis aids in diagnosis ( - Fig. 4). Epiploic appendagi-

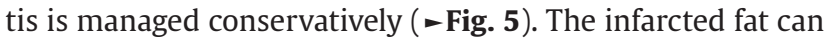
detach, calcify, and becomes a loose peritoneal body. ${ }^{5}$ This can mimic calcified peritoneal deposits on imaging. Mobility on serial scans and no size change differentiates it from the metastatic deposits (-Fig. 6).

\section{Perigastric Appendagitis}

The fatty appendages attached to the gastric wall behave in the same manner as epiploic appendages. A less recognized pathology with only one larger study in literature. ${ }^{9}$ Though the management does not differ much from omental infarct, recognition is key to avoid false interpretations and unnecessary patient anxiety. It is seen as focal fat stranding with gastric attachment commonly related to the greater curvature ( - Fig. 7).

\section{Omental Infarct}

Greater omentum is a double layer of peritoneum containing fat, vessels, and lymphatics. It connects the greater curvature of stomach and transverse colon forming a protective sheet in the anterior abdomen..$^{10}$ On imaging, greater omentum has variable thickness of fat layer anterior to the stomach and colon ( - Fig. 8). Greater omentum acts as a disease limiter and hence commonly involved in peritoneal disease such as infection and carcinomatosis. Diseased omentum on imaging
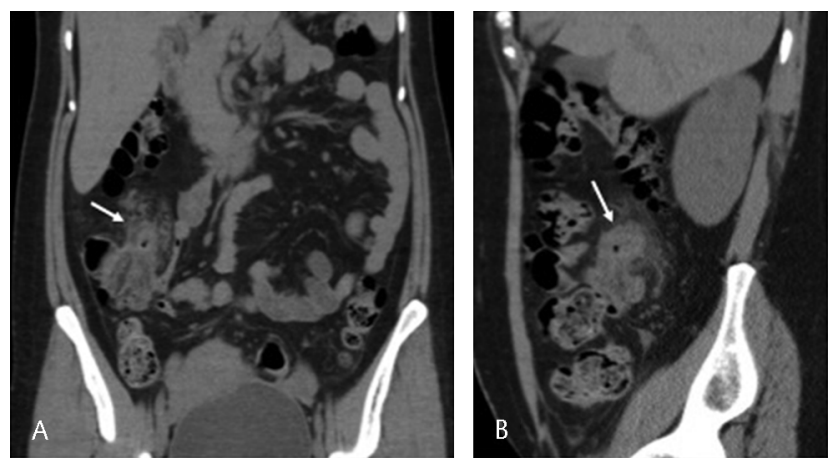

Fig. 4 Coronal (A) and sagittal (B) plain CT images show a solitary caecal diverticulum (arrow) with extensive peridiverticular inflammation resembling epiploic appendagitis. Outpouching and proportionate thickening of the same points toward diverticulitis. CT, computed tomography.
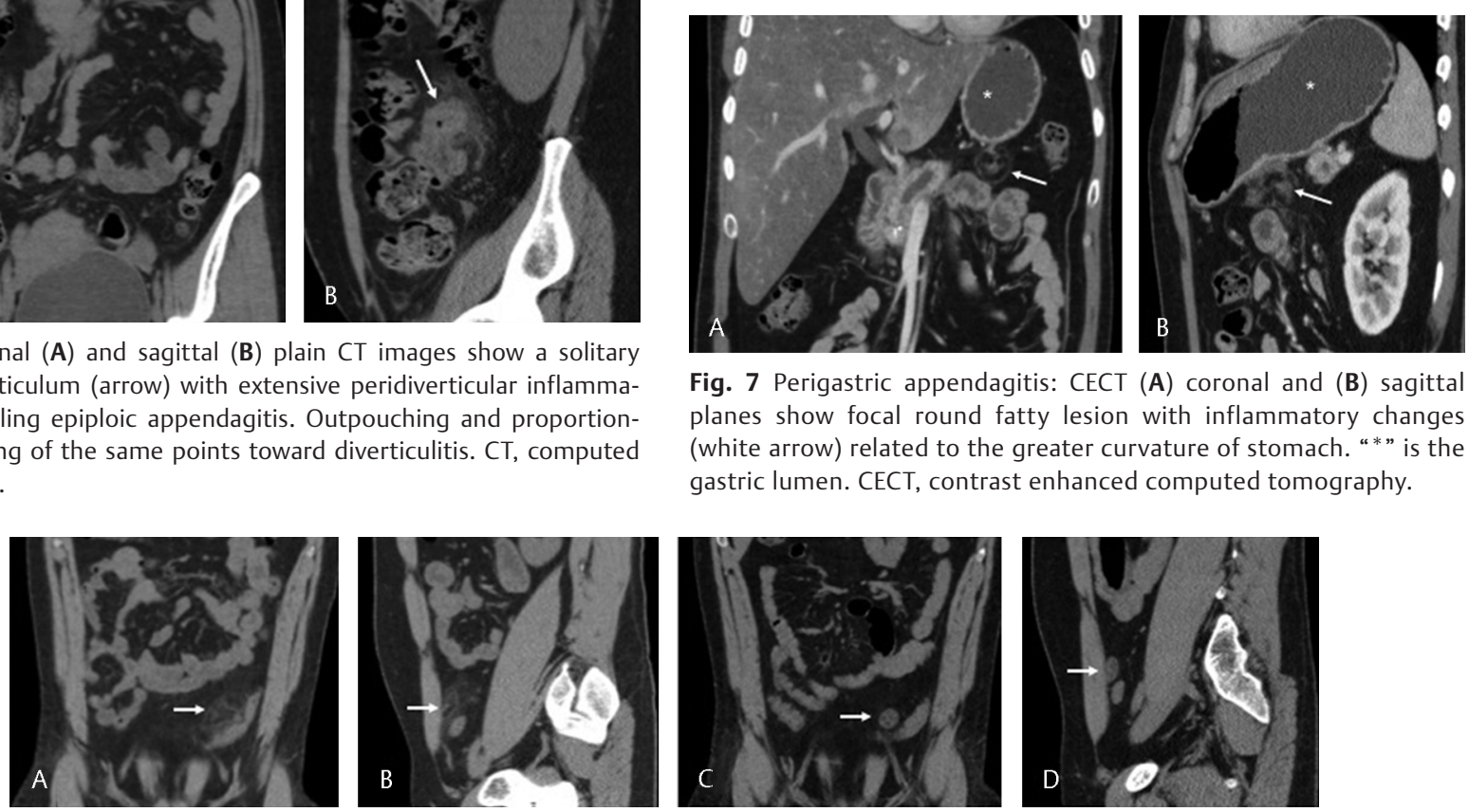

Fig. 7 Perigastric appendagitis: CECT (A) coronal and (B) sagittal planes show focal round fatty lesion with inflammatory changes (white arrow) related to the greater curvature of stomach. "* " is the gastric lumen. CECT, contrast enhanced computed tomography.

Fig. 5 (A, B) Evolution of epiploic appendagitis: plain CT in coronal and sagittal planes at the time of presentation shows inflamed sigmoid epiploicae (arrows). (C, D) Scan done 5 months later shows resolved inflammation and detached fat which may later calcify (arrows). CT, computed tomography. 

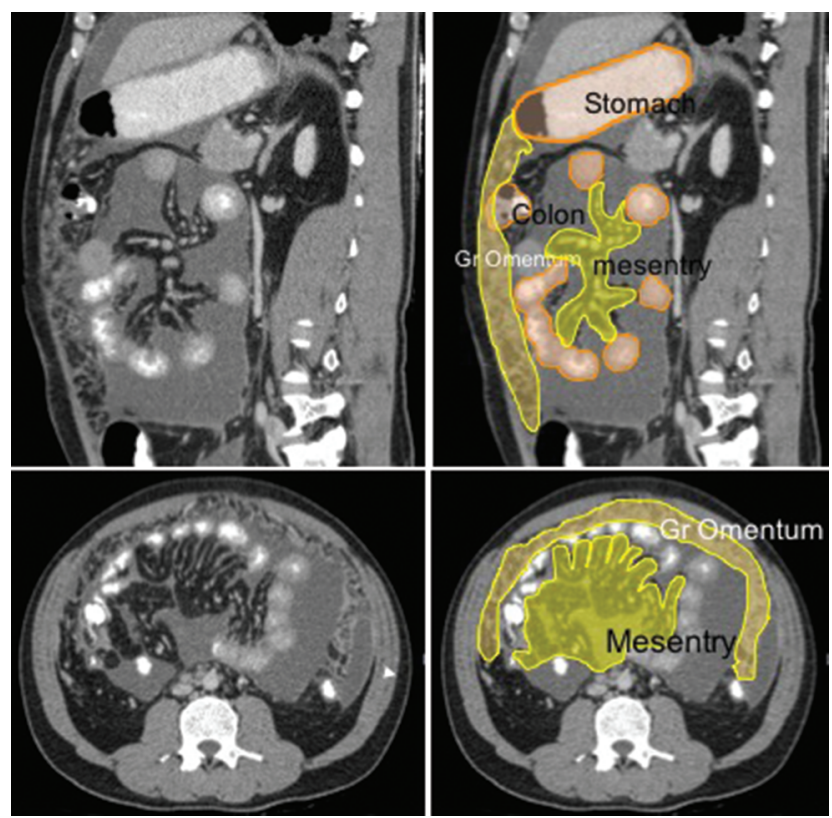

Fig. 8 Sagittal and axial CT: well demarcates the anterior sheet of omentum and mesentry affected by disease process (tuberculosis in this case). CT, computed tomography.

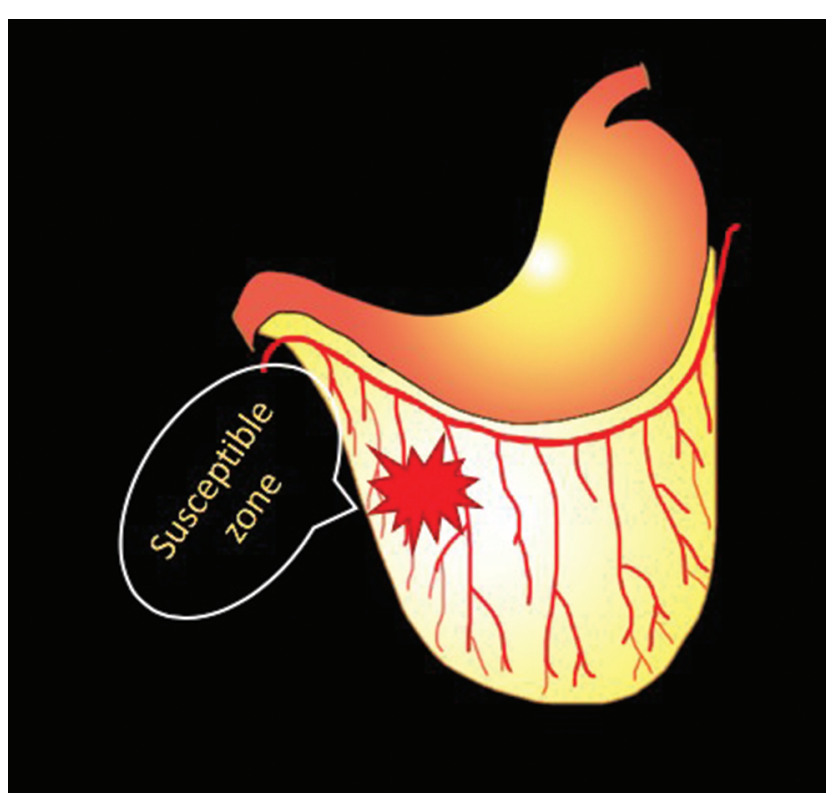

Fig. 9 Illustration depicting the susceptible zone in greater omentum supplied by epiploic arteries branches of right and left gastroepiploic arteries.

up due to superficial location. Use of high resolution linear USG probe is recommended in such clinical scenarios. CT shows focal omental fat stranding with or without swirling of vessels commonly on right side in primary forms ${ }^{14-16}$ (-Figs. 10, 11). Studies show ${ }^{10}$ that secondary infarcts are relatively well defined and denser on CT ( - Fig. 12). Complicated infarcts include encapsulated necrosis or formation of infected collections. ${ }^{2}$

Lesser omentum consists of gastrohepatic and hepatoduodenal ligaments and connects the lesser curvature of the stomach and proximal duodenum with the liver ( - Fig. 13).
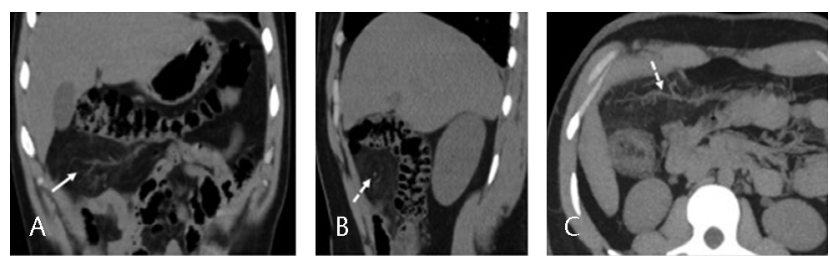

Fig. 10 Primary omental infarct in a young athlete with plain $C T$ in coronal (A), sagittal (B) and axial MIP (C) images revealing large area of fat inflammation (arrow) with central vessel (dashed arrow). $\mathrm{CT}$, computed tomography; MIP, maximum intensity projection.
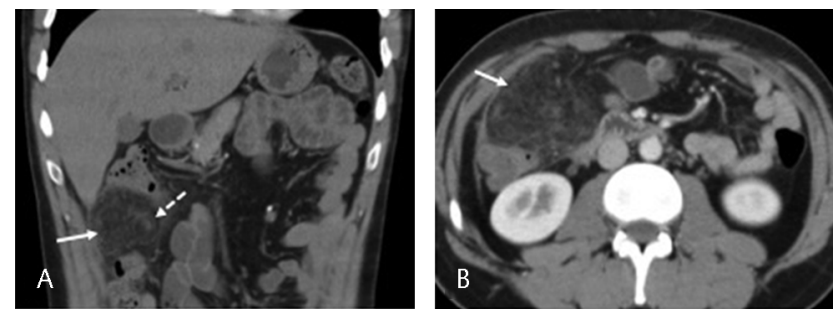

Fig. 11 Coronal (A) and axial (B) CECT images show large area of greater omental infarct with torsion (white arrow) in a young male who presented with sudden onset abdominal pain. Note the central vessel (dashed arrow). CECT, contrast enhanced computed tomography.
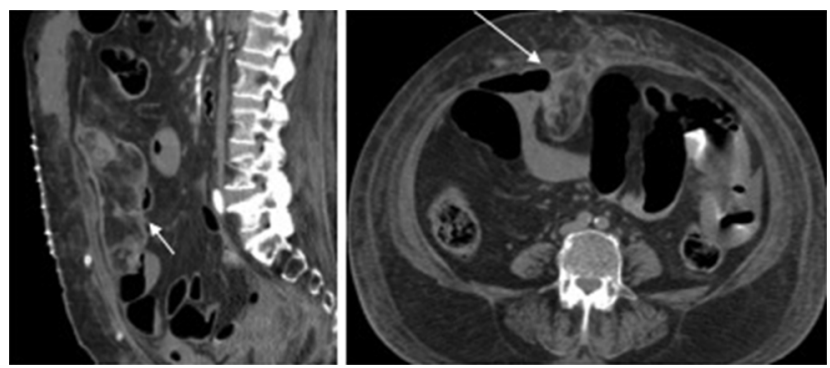

Fig. 12 Secondary omental infarct in postlaparoscopic mesh repair. Compared with primary infarct, the secondary infarct is well defined, mass like (arrows).
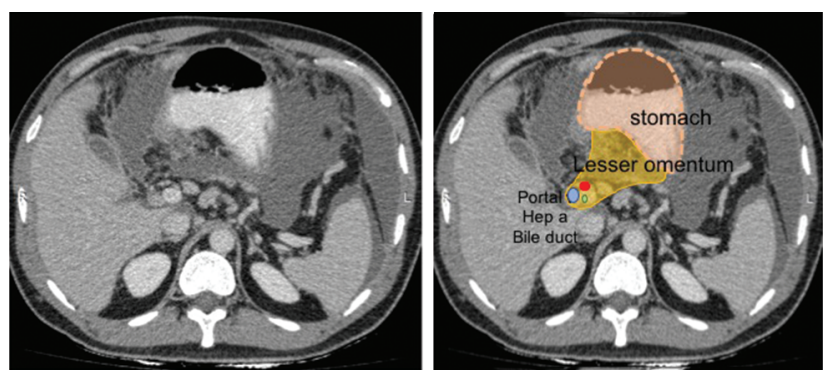

Fig. 13 Imaging illustration of anatomy of lesser omentum.

Unlike greater omentum, lesser omentum is affected predominantly by inflammations involving pancreas, gallbladder, or stomach. Lesser omentum is an uncommon site of infarct and is usually related to pancreatic inflammation, gastric ulcer perforation, etc. Sometimes this entity is used interchangeably with perigastric appendagitis. ${ }^{9}$ Imaging findings are similar to greater omental infarct except for the typical location ( $\boldsymbol{- \text { Fig. }}$ 14). It is also imperative to rule out abnormalities in pancreas, gallbladder, and stomach in such cases before calling it a primary abnormality. Omental infarct is conservatively managed. Surgical exploration preferably 

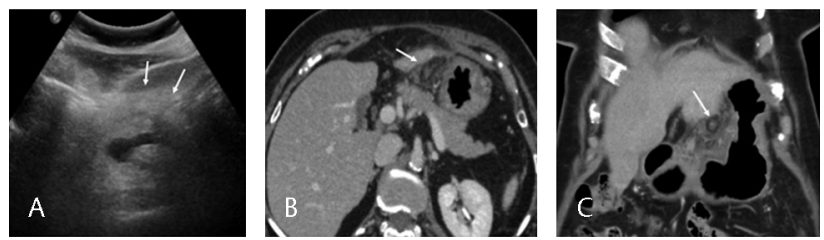

Fig. 14 A 69-year-old female with abdominal pain. (A) USG epigastric region shows focal echogenicity (arrows). (B, C) CECT axial and coronal sections show linear fatty inflammation in lesser omentum (gastro hepatic) with classical central vessel (arrows). Note normal appearing pancreas, gallbladder and stomach in keeping with primary abnormality. CECT, contrast enhanced computed tomography; USG, ultrasonography.

Table 2 Differentiating features between primary and secondary omental infarct

\begin{tabular}{|l|l|}
\hline Primary omental infarct & Secondary omental infarct \\
\hline $\begin{array}{l}\text { In obesity, marathon runners, } \\
\text { hypercoagulable states, and } \\
\text { anatomical variations of the } \\
\text { omentum }\end{array}$ & Direct trauma, surgery \\
\hline Usually right sided & Depends on the site of trauma \\
\hline $\begin{array}{l}\text { Ill-defined with or without } \\
\text { vessel swirling }\end{array}$ & Well defined and denser \\
\hline
\end{tabular}

Table 3 Differentiating features between omental infarct and epiploic appendagitis

\begin{tabular}{|l|l|}
\hline Omental infarct & Epiploic appendagitis \\
\hline Usually right sided (primary) & $\begin{array}{l}\text { Commonly left sided (sigmoid } \\
\text { colon) }\end{array}$ \\
\hline $\begin{array}{l}\text { Ill-defined focal (oval) fat } \\
\text { stranding }\end{array}$ & $\begin{array}{l}\text { Linear fat stranding with cen- } \\
\text { tral vessel (central dot sign), } \\
\text { hyperattenuating ring sign }\end{array}$ \\
\hline $\begin{array}{l}\text { Evolution: resolve completely/ } \\
\text { encapsulated fat necrosis/sec- } \\
\text { ondary infection }\end{array}$ & $\begin{array}{l}\text { Resolve completely/detach } \\
\text { and form loose bodies with or } \\
\text { without calcification }\end{array}$ \\
\hline
\end{tabular}

laproscopic omental necrosectomy is recommended when symptoms are deteriorating. ${ }^{17,18}$ - Tables 2 and $\mathbf{3}$ summarize differences between primary and secondary omental infarct and between omental infarct and epiploic appendagitis respectively.

\section{Encapsulated Fat Necrosis}

Encapsulated necrosis was first described in the breast and mediastinum. The fat degeneration that is secondary to trauma or ischemic insult develops fibrotic capsule which can calcify. ${ }^{19,20}$ Fat density lesion with well encapsulated enhancing wall is seen on imaging (-Fig. 15). The capsule which may enhance with contrast is the hallmark of encapsulated fat necrosis. This appearance mimics liposarcoma and can be differentiated by its noninvasive nature, tenderness on palpation, and either static or progressive decrease in size of the lesion on follow-up (-Fig. 16). Liposarcomas are commonly retroperitoneal and hence location can be used to differentiate.
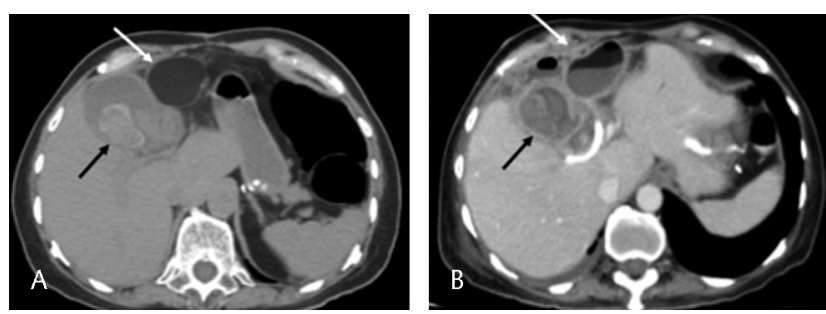

Fig. 15 CT axial sections reveal encapsulated fat necrosis in a patient seen as a well defined fat density with smooth wall around (white arrow). Note calculus cholecystitis (A) (black arrow) which eventually fistulized with the fat necrosis later (B). CT, computed tomography.
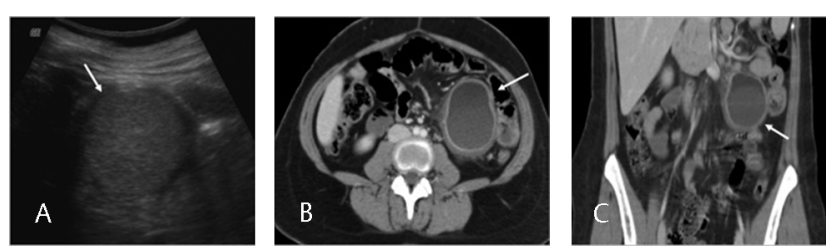

Fig. 16 Another case of encapsulated fat necrosis appearing uniformly echogenic on USG (A), showing smooth, thick enhancing wall (arrows) with mild surrounding inflammation on CECT $(\mathbf{B}, \mathbf{C})$. Initially misdiagnosed as mesentric cyst. CECT, contrast enhanced computed tomography; USG, ultrasonography.

\section{Torsion of Fatty Appendage of Falciform Ligament}

Falciform ligament is a double fold of peritoneum that divides the right and left lobes of liver. It courses from superior edge of the liver to the inferior border of the diaphragm. Contents include ligamentum teres, paraumbilical veins, and varying amount of extraperitoneal fat ( - Fig. 17). Some consider falciform fat as part of perigastric appendage. This fatty appendage can undergo torsion and can present with acute epigastric pain. Knowledge of the anatomy is important to recognize this entity ( - Fig. 18). On CT, focal fat inflammation with a central vessel seen at the expected level of falciform ligament. Tenderness can be elicited on USG. ${ }^{21-24}$

\section{Secondary Fat Saponification}

Pancreatitis is the commonest cause of secondary fat saponification $^{2,25}$ followed by long-term steroid therapy. ${ }^{26}$ Inflammation of pancreas releases lipolytic enzymes that cause fat digestion and necrosis. Retroperitoneal and mesentric fat are predominantly involved. Fat necrosis after the resolution of acute phase are seen as nodular lesions which may be scattered or confluent ( $\mathbf{- F i g . ~ 1 9 )}$ ). These nodules can enhance and greatly mimic peritoneal carcinomatosis or tubercular peritonitis. ${ }^{25}$ Awareness of this entity along with history of pancreatitis can help in diagnosis.

\section{Metabolic, Reparative, and Responsive Causes}

Alteration in behavior of fat as a result of metabolic imbalances or as a reparative response results in either hypertrophy or dystrophy. For completion sake, Dercam's disease, a metabolic condition characterized by multiple lipomas is also included in the discussion. 

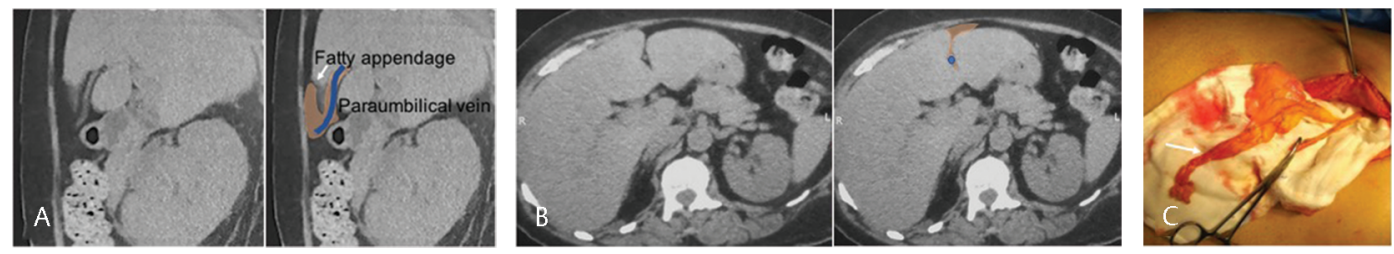

Fig. 17 (A, B) Imaging anatomy of falciform ligament region. Orange is the fat and blue is the paraumbilical vein. (C). Intraoperative image showing umbilical vein (held by artery forceps) and fatty appendage (arrow).
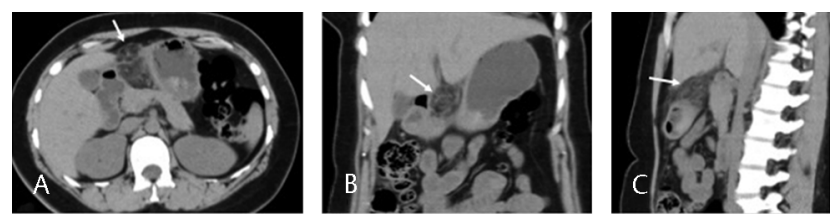

Fig. 18 Axial (A), coronal (B) and sagittal (C) plain CT of a young female with history of child kick with anterior abdominal pain shows inflammation/torsion of the fatty appendage of falciform ligament (white arrow). CT scan done after 2 weeks (not shown) showed spontaneous resolution. $\mathrm{CT}$, computed tomography.
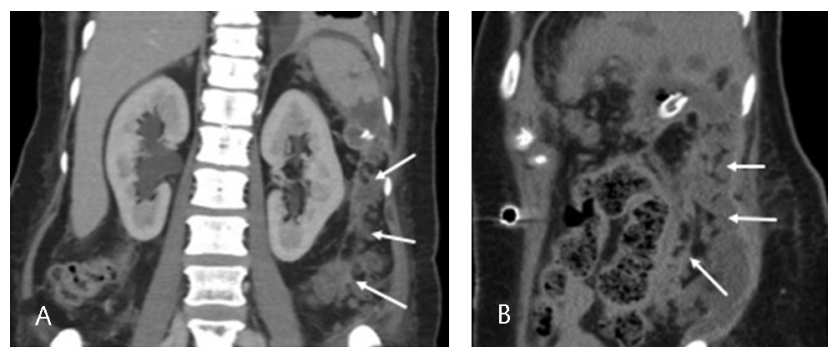

Fig. 19 CECT in coronal (A) and parasagittal plane (B) shows diffuse retroperitoneal fat necrosis manifesting as hypo enhancing nodular soft tissue (white arrows) in a patient with necrotizing pancreatitis.

\section{Lipohypertrophy}

Repeated insulin injections (type $1>2$ diabetes) in the subcutaneous tissue (commonly anterior abdominal wall) causes focal fat proliferation referred as lipohypertrophy or "insulin balls." Lipohypertrophy leads to reduced local pain sensation and hence patients prefer injections at same site leading to a vicious cycle. It is postulated that lipohypertrophy impairs insulin absorption and hence leads to insensitivity. Local amyloid deposition at the injection site is another cause of impaired insulin sensitivity. Lipohypertrophy is usually diagnosed by palpation and appears as an amphibolic and lobular growth, whereas amyloid tumors are more solid and firm. Lipohypertrophy generally regresses soon after cessation of insulin injection, whereas localized amyloidosis does not. Lipohypertrophy mimics liposarcoma, but history and location can aid in differentiation with confounding cases requiring biopsy. Imaging shows altered, hypertrophied fat at sites of affection $(\boldsymbol{- F i g . ~ 2 0})^{2,27}$

\section{Lipomatosis}

Fat proliferation with unaltered adipose tissue can be generalized or focal. Generalized fat deposition in abdomen is referred to as "abdominal or pelvic lipomatosis" ( - Fig. 21). Excess fat can compress or stretch urinary bladder and
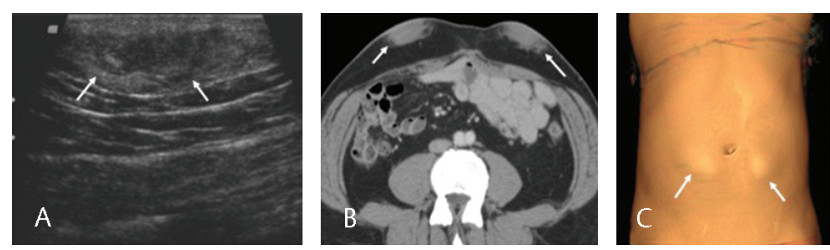

Fig. 20 Focal anterior abdominal fat echogenicity on USG $(\mathbf{A})$ in a diabetic on insulin injections. CECT in axial plane (B) shows symmetrical areas of soft tissue (arrows) replacing fat at the site of insulin injection suggestive of lipodystrophy. Note symmetrical abdominal wall bulge on VRT (C) referred to as "insulin balls." CECT, contrast enhanced computed tomography; USG, ultrasonography; VRT, volume rendering technique.
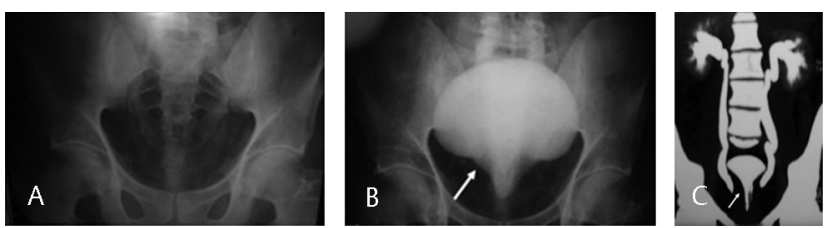

Fig. 21 (A) Plain radiograph of pelvis shows increased lucency. (B) Intravenous urogram reveals typical "inverted tear drop" bladder (arrow). (C) MIP coronal CT shows the same finding. CT, computed tomography, MIP, maximum intensity projection.
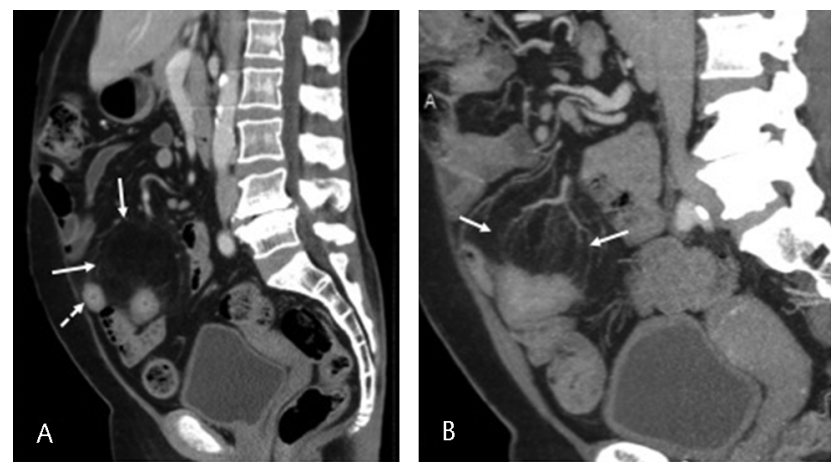

Fig. 22 Sagittal CECT (A) with MIP (B) shows focal fat (white arrow) in a patient with active Crohn's disease. Dashed arrow shows bowel wall thickening and lumen narrowing. Also note prominent vasa recta in MIP image. CECT, contrast enhanced computed tomography; MIP, maximum intensity projection.

rectum causing characteristic signs, such as "inverted tear drop," in the former. ${ }^{28,29}$ Focal fat proliferations are secondary to inflammation and commonly seen as "creeping fat" sign in inflammatory bowel disease ( - Fig. 22). ${ }^{29}$

\section{Lipodystrophy}

Lipodystrophies are rare conditions which are either familial or acquired, partial, or generalized. ${ }^{30,31}$ Congenital 
causes are autosomal recessive with characteristic facies. Acquired causes commonly is HIV related or drug induced. Variable atropy of intra-abdominal, retroperitoneal, subcutaneous, and intermuscular fat is noted in imaging. MRI is commonly used as ancillary test. Acquired causes spare peritoneal and mediastinal fat with predominant subcutaneous atrophy in contrast to congenital lipodystrophy (-Fig. 23).

\section{Dercum's Disease}

Also referred to as adiposis dolorosa, it is not an uncommon disorder presenting with multiple painful lipomas. It can be associated with weakness, endocrine, lipid abnormalities, and mental disturbances. It can be sporadic or autosomal dominant. Trunk and legs are common locations. ${ }^{32}$ Imaging features are similar to the usual lipoma but differ in being multiple, $<2 \mathrm{~cm}$, painful, confined only to subcutaneous plane with no surrounding inflammation (-Fig. 24). Differentials include localized lymphedema and cellulitis. Presence of skin thickening favors lymphedema. Cellulitis had relatively extensive involvement and shows contrast enhancement unlike lipomas in Dercum's disease. ${ }^{33}$

\section{Misty Mesentry Spectrum}

Haziness (regional increase in fat density) in mesentry is a very nonspecific finding on CT with many systemic diseases causing it. It shows strong association with concurrent

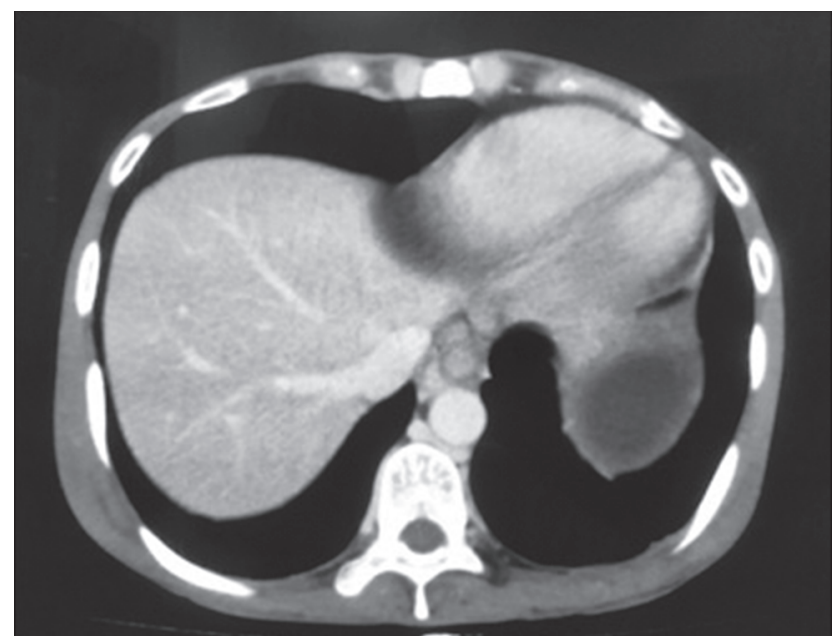

Fig. 23 Diffuse subcutaneous fat atrophy in a patient with HIV infection on HAART regimen. HAART, highly active anti retroviral therapy. malignancies and other fibrosing conditions such as retroperitoneal fibrosis and pseudotumor. Increase in fat density may be secondary to edema or fluid, hemorrhage, fibrosis, necrosis, or tumor. ${ }^{34,35}$ Mesentric panniculitis is an umbrella term comprising of three disease stages, mesentric lipodystrophy, panniculitis, and sclerosing mesenteritis. ${ }^{36}$ Mesentric lipodystrophy is a histopathology term and imaging is usually normal. Mesentric panniculitis shows increase in mesentric fat density with "tumoral pseudocapsule" sign which refers to a thin $(<3 \mathrm{~mm})$ peripheral curvilinear band of soft tissue attenuation separating from the normal fat ( $50 \%$ sensitivity) and "perinodal and perivascular fat sparing" with $75 \%$ sensitivity (-Fig. 25). ${ }^{34,37}$ Sclerosing mesentritis also known as retractile mesentritis is a chronic inflammation of the mesentry predominated by fibrosis. On CT, it is commonly seen as a soft tissue mass within the mesentry with infiltrative margins and tethering (-Fig. 26). Calcification and cyst formation can be seen. There may be perivascular fat sparing, but encasement of vessels is also reported due to fibrosis. ${ }^{38}$ Imaging differentials include lymphoma, carcinoid, and desmoid tumor.
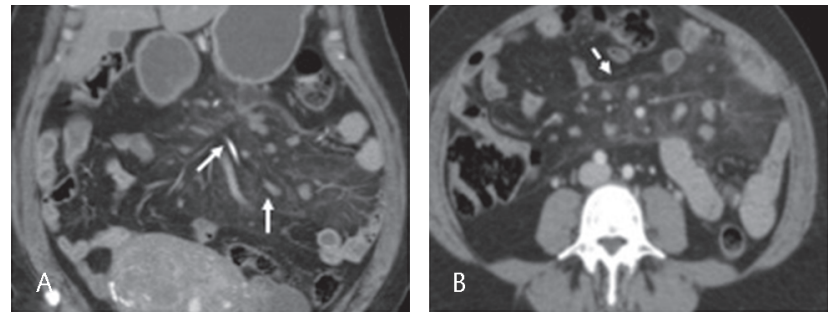

Fig. 25 (A, B) CECT in coronal and axial planes show classical "mesentric panniculitis" with typical perinodal, perivascular sparing (solid arrows) and thin capsule (broken arrow). CECT, contrast enhanced computed tomography.
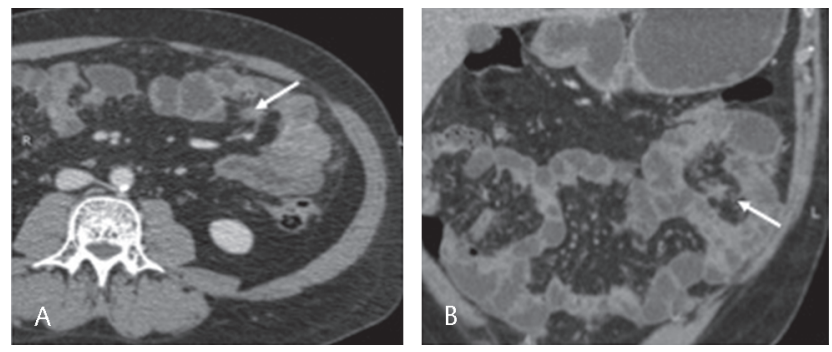

Fig. 26 CECT in axial (A) and coronal (B) planes shows central soft tissue with desmoplasia tethering the small bowel (arrow)-sclerosing mesenteritis in a patient with nonspecific abdominal pain. CECT, contrast enhanced computed tomography.
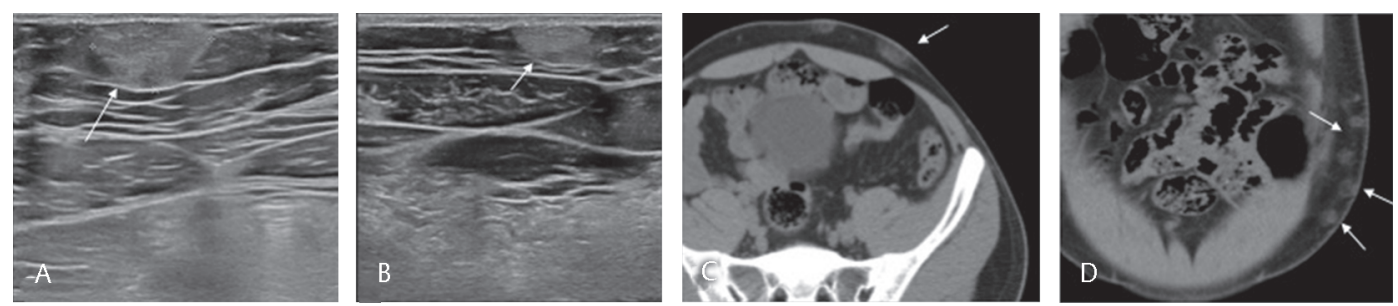

Fig. 24 USG (A, B) of anterior abdomen shows multiple echogenic subcutaneous lesions (arrows). Corresponding CT images in axial (C) and coronal planes (D) show multiple ill-defined hypodense areas in abdominal wall, consistent with Dercam's disease. CT, computed tomography; USG, ultrasonography. 


\section{Infections}

Tuberculosis has predilection for peritoneal involvement. Imaging features on CT include a "wet type," which is characterized by ascites; a "fibrotic type," which manifests as large omental and mesenteric masses; and a "dry type," seen as diffuse, fibrous peritoneal thickening. ${ }^{39,40}$ Presence of necrotic lymphadenopathy, calcified nodes, and chest tuberculosis may be seen with any form and helps in diagnosis (-Fig. 27). Thin omental line covering the infiltrated omentum is somewhat specific for tubercular peritonitis. ${ }^{41}$

\section{Neoplasms}

A detailed description of all neoplasms of fat origin is beyond the scope of this article. Neoplasms of primary fat origin include benign lesions such as lipoma, hibernoma, and malignant counterpart, liposarcoma. Liposarcomas are commonly retroperitoneal in location. Tumors like desmoid, carcinoid can arise from mesentry and mimic retractile mesentritis. Malignant neoplasms with preferential involvement of abdominal fat include mesothelioma, lymphoma, and peritoneal carcinomatosis (-Fig. 28). Peritoneal carcinomatosis can greatly mimic omental infarct and tubercular peritonitis and careful search for primary malignancy, such as ovary, stomach, colon, is mandated. ${ }^{41-43}$

\section{Conclusion}

Abdominal fat can undergo inflammation and necrosis in variety of conditions and presents with acute abdomen.
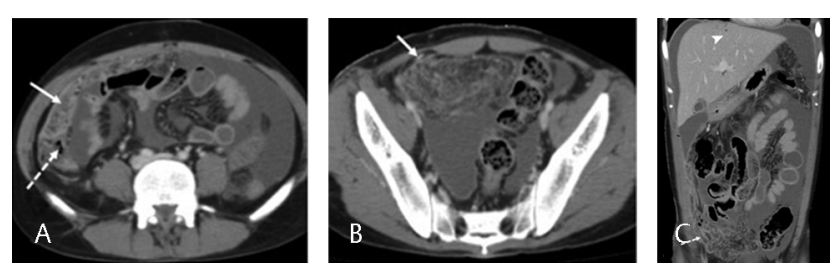

Fig. 27 CECT axial (A, B) and coronal (C) sections show diffuse omental thickening with nodularity (white arrow), ascites in a patient with abdominal pain, fever and weight loss-biopsy showed caseating granulomas c/w tuberculosis. Air foci within the omentum (dashed arrow) and portal venous gas (arrowhead) is secondary to biopsy. CECT, contrast enhanced computed tomography.

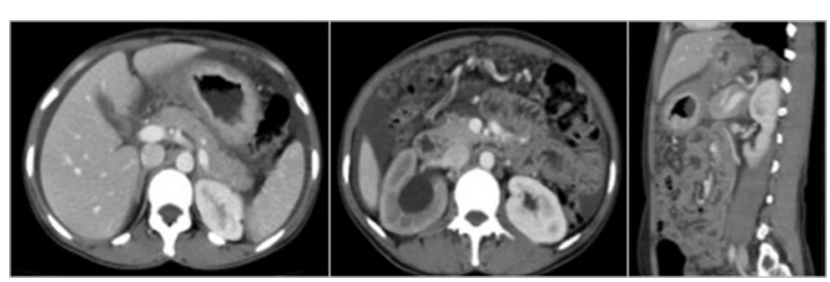

Fig. 28 Gastric adenocarcinoma with peritoneal carcinomatosis. Note diffuse gastric wall thickening, ascites and omental nodularity.
Imaging features (USG and CT) are typical in most circumstances. Secondary saponification of fat is commonly encountered in pancreatitis. Metabolic and reparative fat changes manifest as proliferation and dystrophy. Some neoplasms show preferential fat involvement and should be differentiated from more benign conditions like tubercular peritonitis. A detailed clinical history is always mandated in accurate diagnosis.

\section{Conflict of Interest}

None declared.

\section{References}

1 Ruess L, Frazier AA, Sivit CJ. CT of the mesentery, omentum, and peritoneum in children. Radiographics 1995;15(1):89-104

2 Kamaya A, Federle MP, Desser TS. Imaging manifestations of abdominal fat necrosis and its mimics. Radiographics 2011;31(7):2021-2034

3 Eloi JC, Epifanio M, de Gonçalves MM, et al. Quantification of abdominal fat in obese and healthy adolescents using 3 tesla magnetic resonance imaging and free software for image analysis. PLoS One 2017;12(1):e0167625

4 Sideras PA, Pat V, Li Z, Heiba S, Kostakoglou L. PET-CT of mesenteric panniculitis: a potential confounder of lymphoma. Radiography 2015;21(1):e51-e53

5 Singh AK, Gervais DA, Hahn PF, Sagar P, Mueller PR, Novelline RA. Acute epiploic appendagitis and its mimics. Radiographics 2005;25(6):1521-1534

6 Chakraborty AK, Olcott EW, Jeffrey BR. Hyperechoic abdominal fat: a sentinel sign of inflammation. Ultrasound Q 2019; 35(2):186-194

7 Giambelluca D, Cannella R, Caruana G, et al. CT imaging findings of epiploic appendagitis: an unusual cause of abdominal pain. Insights Imaging 2019;10(1):26

8 Nadida D, Amal A, Ines M, et al. Acute epiploic appendagitis: Radiologic and clinical features of 12 patients. Int J Surg Case Rep 2016;28:219-222

9 Justaniah AI, Scholz FJ, Katz DS, Scheirey CD. Perigastric appendagitis: CT and clinical features in eight patients. Clin Radiol 2014;69(12):e531-e537

10 Sompayrac SW, Mindelzun RE, Silverman PM, Sze R. The greater omentum. AJR Am J Roentgenol 1997;168(3):683-687

11 Pereira JM, Sirlin CB, Pinto PS, Jeffrey RB, Stella DL, Casola G. Disproportionate fat stranding: a helpful CT sign in patients with acute abdominal pain. Radiographics 2004;24(3):703-715

12 Choh NA, Shera TA, Jabeen S, et al. Primary and secondary omental infarction: A 5-year experience in a tertiary care hospital. Saudi Surgical J 2017;5(2):77-81

13 Occhionorelli S, Zese M, Cappellari L, Stano R, Vasquez G. Acute abdomen due to primary omental torsion and infarction. Case Rep Surg 2014;2014:208382

14 Devos H, Goethals L, Belsack D, et al. Fat misbehaving in the abdominal cavity: a pictorial essay. Pol J Radiol 2020; 85:e32-e38

15 Udechukwu NS, D'Souza RS, Abdulkareem A, Shogbesan O. Computed tomography diagnosis of omental infarctionpresentingasanacuteabdomen.RadiolCaseRep2018; 13(3):583-585

16 Singh AK, Gervais DA, Lee P, et al. Omental infarct: CT imaging features. Abdom Imaging 2006;31(5):549-554

17 Soobrah R, Badran M, Smith SG. Conservative management of segmental infarction of the greater omentum: a case report and review of literature. Case Rep Med 2010;2010:765389 
18 Barai KP, Knight BC. Diagnosis and management of idiopathic omental infarction: a case report. Int J Surg Case Rep 2011;2(6):138-140

19 De Kock I, Delrue L. Encapsulated mesenteric fat necrosis. J Belg Soc Radiol 2016;100(1):53

20 Bhatt MY, Martínez-Jiménez S, Rosado-de-Christenson ML, Watson KR, Walker CM, Kunin JR. Imaging manifestations of mediastinal fat necrosis. Case Rep Radiol 2013;2013:323579

21 Vanderschueren L, Coulier B. Focal fat infarction of the falciform ligament: typical CT appearance of a rare form of intra-peritoneal focal fatty infarction. J Belg Soc Radiol 2019;103(1):28

22 Aggarwal A, Aggarwal R, Prabakaran M. Intraabdominal focal fat infarction due to torsion of falciform ligament appendage. Sch J App Med Sci 2018;6(10):4123-4125

23 Indiran V, Dixit R, Maduraimuthu P. Unusual cause of epigastric pain: intra-abdominal focal fat infarction involving appendage of falciform ligament - case report and review of literature. GE Port J Gastroenterol 2018;25(4):179-183

24 Uyttenhove F, Leroy C, Nzamushe Lepan Mabla JR, Ernst $\mathrm{O}$. Torsion of a fatty fringe of the falciform ligament, a rare cause of right hypochondrial pain. Diagn Interv Imaging 2013; 94(6):637-639

25 Smith JP, Arnoletti JP, Varadarajulu S, Morgan DE. Postpancreatitis fat necrosis mimicking carcinomatosis. Radiol Case Rep 2015;3(2):192

26 Gupta P, Bhattatiry M, Eshaghi N, Tirkes A, Kumar M. Idiopathic retroperitoneal fat necrosis in a patient on long-term steroids. The Internet J Radiology 2004;4(1):x

27 Abu Ghazaleh H, Hashem R, Forbes A, et al. A systematic review of ultrasound-detected lipohypertrophy in insulin-exposed people with diabetes. Diabetes Ther 2018;9(5):1741-1756

28 Smereczyński A, Kołaczyk K, Bernatowicz E. Intra-abdominal fat. Part I. The images of the adipose tissue localized beyond organs. J Ultrason 2015;15(62):318-325

29 Smereczyński A, Kołaczyk K, Bernatowicz E. Intra-abdominal fat. Part II: non-cancerous lesions of the adipose tissue localized beyond organs. J Ultrason 2016;16(64):32-43

30 Premkumar A, Chow C, Bhandarkar P, et al. Lipoatrophiclipodystrophic syndromes: the spectrum of findings on MR imaging. AJR Am J Roentgenol 2002;178(2):311-318
31 Garg A. Acquired and inherited lipodystrophies. N Engl J Med 2004;350(12):1220-1234

32 Tins BJ, Matthews C, Haddaway M, et al. Adiposis dolorosa (Dercum's disease): MRI and ultrasound appearances. Clin Radiol 2013;68(10):1047-1053

33 Petscavage-Thomas JM, Walker EA, Bernard SA, Bennett J. Imaging findings of adiposis dolorosa vs. massive localized lymphedema. Skeletal Radiol 2015;44(6):839-847

34 McLaughlin PD, Filippone A, Maher MM. The "misty mesentery": mesenteric panniculitis and its mimics. AJR Am J Roentgenol 2013;200(2):W116-23

35 Thornton E, Mendiratta-Lala M, Siewert B, Eisenberg RL. Patterns of fat stranding. AJR Am J Roentgenol 2011; 197(1):W1-14

36 McDermott RL, Hutchinson B, Ryan C, et al. Mesenteric lipodystrophy - An unusual intraabdominal mass. Int J Surg Case Rep 2013;4(2):232-234

37 Daskalogiannaki M, Voloudaki A, Prassopoulos P, et al. CT evaluation of mesenteric panniculitis: prevalence and associated diseases. AJR Am J Roentgenol. AJR Am J Roentgenol 2000;174(2):427-431

38 Horton KM, Lawler LP, Fishman EK. CT findings in sclerosing mesenteritis (panniculitis): spectrum of disease. Radiographics 2003;23(6):1561-1567

39 Bonafe T, Nicola R, Kovacs J. Differential considerations for omental fat infiltration and thickening on CT. J Am Osteopath Coll Radiol 2014;3(4)

40 Pickhardt PJ, Bhalla S. Unusual nonneoplastic peritoneal and subperitoneal conditions: CT findings. Radiographics 2005;25(3):719-730

41 Shin NY, Kim MJ, Chung JJ, Chung YE, Choi JY, Park YN. The differential imaging features of fat-containing tumors in the peritoneal cavity and retroperitoneum: the radiologic-pathologic correlation. Korean J Radiol 2010;11(3):333-345

42 Ha HK, Jung JI, Lee MS, et al. CT differentiation of tuberculous peritonitis and peritoneal carcinomatosis. AJR Am J Roentgenol 1996;167(3):743-748

43 Smereczyński A, Kołaczyk K, Bernatowicz E. Intra-abdominal fat. Part III. Neoplasms lesions of the adipose tissue. J Ultrason 2016;16(65):145-154 